\title{
User access to Cryo-EM at EMSL: Opportunities Linking Omics and Structural Biology
}

Trevor Moser ${ }^{1}$, Irina Novikova ${ }^{1}$, Amar Parvate ${ }^{1}$, Samantha Powell ${ }^{2}$ and James Evans ${ }^{1}$

${ }^{1}$ Pacific Northwest National Laboratory, United States, ${ }^{2}$ Pacific Northwest National Laboratory, Richland, Washington, United States

The Environmental Molecular Sciences Laboratory (EMSL), a Department of Energy funded national user facility, recently acquired and commissioned a new Krios G3i cryo-TEM and Aquilos 2 cryo-FIB/SEM to expand the available electron microscopy capabilities for biological, environmental and materials research.

The Krios G3i microscope has complete screening, data collection and image processing workflows for: 1) micro-electron diffraction of small molecule or protein crystals, 2) single particle analysis of soluble and membrane protein complexes and 3) electron tomography of whole cells or isolated organelles. It is equipped with a K3 direct electron detector, Ceta-D camera, phase plate and Bioquantum energy filter. In addition to semi-automated data collection, we have installed automated image processing workflows for real-time monitoring feedback of session quality and full 3D reconstruction of all workflows. To date we have demonstrated sub-2 angstrom 3D reconstructions for single particle and micro-electron diffraction workflows and sub-nanometer resolution for whole cell tomography. The Aquilos 2 cryo-FIB/SEM is fully equipped for automated cryo-slice-and-view, site-selective lift-out and automated batch lamella generation. This allows for pseudo-3D imaging of thick samples as well as thinning of thick samples to make them compatible on the Krios G3i for tomography and micro-electron diffraction applications. While we can provide very rapid access for samples that arrive pre-frozen on clipped and pre-screened grids, we can also begin with samples that arrive in buffer and require all steps of the cryo-EM workflow. In a subset of cases, we can also start from a provided gene of interest and employ or cell-free expression system to produce enough protein for structural characterization.

Due to the joint funding approach used to acquire the instruments, multiple avenues exist for users to access these capabilities. Proposal submission to normal EMSL user proposal calls permits combining cryo-EM with other capabilities at EMSL - such as flow cytometry, native mass spectrometry, superresolution fluorescence microscopy or single-cell omics. For project only seeking cryo-EM access, an expedited submission and review process is available. This presentation will provide an overview of the available capabilities as well as highlight the various access mechanisms and user science performed to date.

Funding statement: Pacific Northwest National Laboratory is operated by Battelle for the U.S. Department of Energy under Contract DE-AC05-76RL01830. This program is supported by the U. S. Department of Energy, Office of Science, through the Genomic Science Program, Office of Biological and Environmental Research, under FWPs 74194 and 74195. The work was performed at EMSL (grid.436923.9), a DOE Office of Science User Facility sponsored by the Biological and Environmental Research program. 

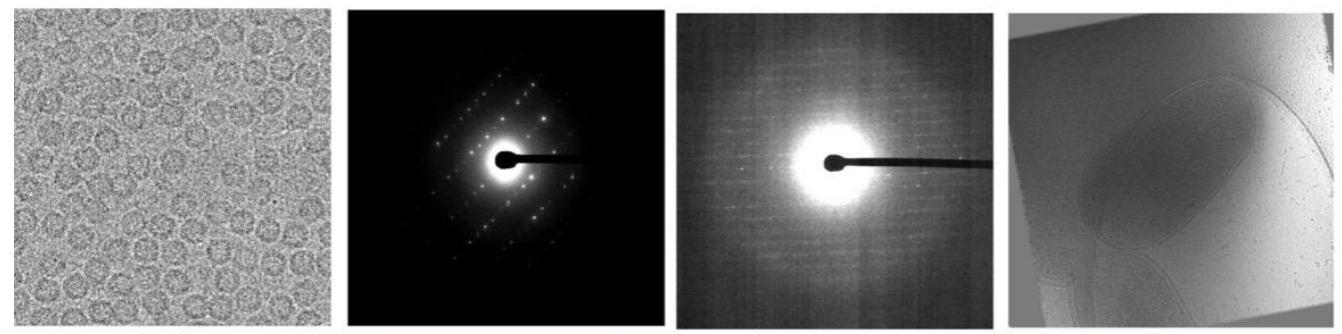

Single Particle Imaging

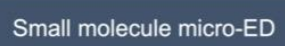

Protein micro-ED

Whole Cell Tomography
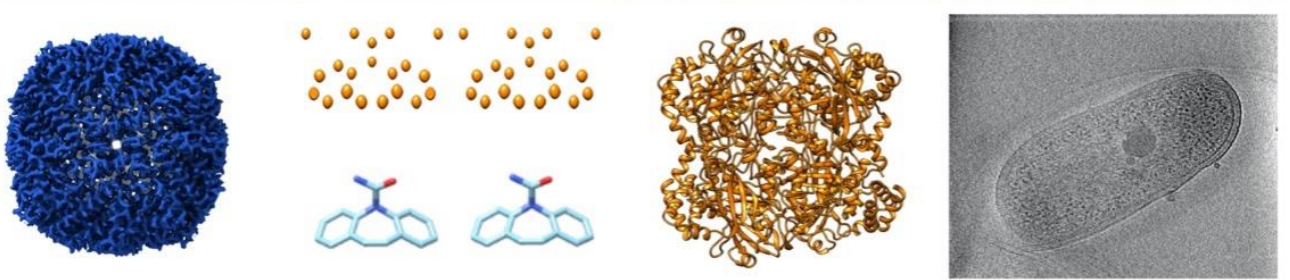

Figure 1. Benchmarking examples of each of the cryo-EM sample workflows available to users on the new Krios G3i microscope at EMSL. Top row shows raw data while bottom row shows reconstructed volumes. 